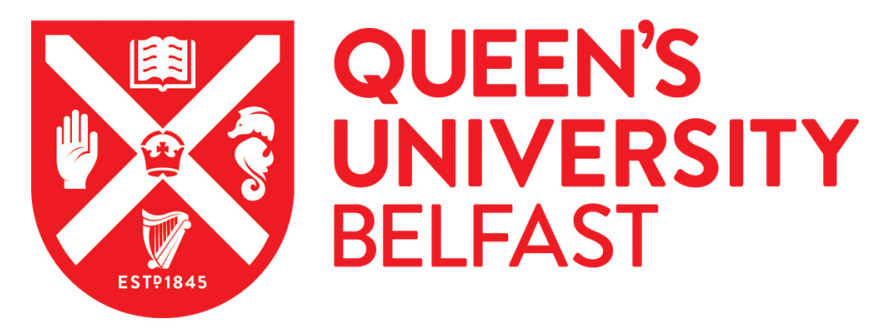

\title{
Positron Binding and Annihilation in Alkane Molecules
}

Swann, A. R., \& Gribakin, G. F. (2019). Positron Binding and Annihilation in Alkane Molecules. Physical Review Letters, 123, [113402]. https://doi.org/10.1103/PhysRevLett.123.113402

Published in:

Physical Review Letters

Document Version:

Publisher's PDF, also known as Version of record

Queen's University Belfast - Research Portal:

Link to publication record in Queen's University Belfast Research Portal

Publisher rights

(c) 2019 American Physical Society. This work is made available online in accordance with the publisher's policies. Please refer to any applicable terms of use of the publisher.

\section{General rights}

Copyright for the publications made accessible via the Queen's University Belfast Research Portal is retained by the author(s) and / or other copyright owners and it is a condition of accessing these publications that users recognise and abide by the legal requirements associated with these rights.

Take down policy

The Research Portal is Queen's institutional repository that provides access to Queen's research output. Every effort has been made to ensure that content in the Research Portal does not infringe any person's rights, or applicable UK laws. If you discover content in the Research Portal that you believe breaches copyright or violates any law, please contact openaccess@qub.ac.uk. 


\title{
Positron Binding and Annihilation in Alkane Molecules
}

\author{
A. R. Swann $\odot^{*}$ and G. F. Gribakin ${ }^{\dagger}$ \\ School of Mathematics and Physics, Queen's University Belfast, University Road, Belfast BT7 1NN, United Kingdom
}

(Received 28 May 2019; revised manuscript received 20 June 2019; published 13 September 2019)

\begin{abstract}
A model-potential approach has been developed to study positron interactions with molecules. Binding energies and annihilation rates are calculated for positron bound states with a range of alkane molecules, including rings and isomers. The calculated binding energies are in good agreement with experimental data, and the existence of a second bound state for $n$-alkanes $\left(\mathrm{C}_{n} \mathrm{H}_{2 n+2}\right)$ with $n \geq 12$ is predicted in accord with experiment. The annihilation rate for the ground positron bound state scales linearly with the square root of the binding energy.
\end{abstract}

DOI: 10.1103/PhysRevLett.123.113402

The ability of positrons to bind to molecules underpins the spectacular phenomenon of resonantly enhanced positron annihilation observed in most polyatomic gases [1]. In this process, the positron is captured by the molecule, its excess energy being transferred into molecular vibrations $[2,3]$. The corresponding annihilation rates depend strongly on the molecular size and display remarkable chemical sensitivity [4-8]. Observation of energy-resolved resonant annihilation [9] has also enabled measurements of the positron binding energy $\varepsilon_{b}$. Binding energies ranging from few to a few hundred of meV have been determined experimentally for over 70 , mostly nonpolar, molecular species, including alkanes, aromatics, partially halogenated hydrocarbons, alcohols, formates, and acetates [10-13].

This body of data is barely understood from a theoretical standpoint, in spite of a long history of the question [14,15]. Nearly all existing calculations of positron binding considered strongly polar molecules, where binding is guaranteed at any level of theory [16]. A variety of methods were used, including Hartree-Fock [19], configuration interaction [20-22], quantum Monte Carlo method [2325], explicitly correlated Gaussians [26], and the anyparticle molecular-orbital approach [27]. The majority of calculations examined simple diatomics, such as alkali hydrides [24] and metal oxides [23], or triatomics: hydrogen cyanide [21,25] and $\mathrm{C} X Y(X, Y=\mathrm{O}, \mathrm{S}, \mathrm{Se})$ [28] (see Ref. [29] for more information). In spite of this effort, of all the molecules studied experimentally, theoretical predictions are available only for five strongly polar species (acetaldehyde, propanal, acetone, acetonitrile, and propionitrile [30-32]), and the best agreement does not exceed $25 \%$ (for acetonitrile, $\varepsilon_{b}=136 \mathrm{meV}$, theory [32], vs $180 \mathrm{meV}$, experiment [11]). Critically, quantum-chemistry calculations have so far failed to predict positron binding to nonpolar molecules with any degree of accuracy [33].

To address this problem, we construct a simple physical model that enables calculations of positron binding to a wide range of polyatomic species and has predictive capability. We apply the model to a range of alkanes and find good agreement with experiment, which confirms that the effective positron-molecule potential is largely "additive" and distributed over the molecule, and that its short-range part is just as important as the long-range behavior determined by the molecular polarizability. While this short-range part cannot be described $a b$ initio with the required accuracy, we show that it can be parametrized in a reliable way. This opens the way for calculating positron binding energies, annihilation rates, and $\gamma$ spectra for all molecules that have been studied experimentally and for making predictions for other molecules. Understanding positron binding to molecules also sheds light on its counterpart- the problem of electron attachment to molecules and formation of molecular anions.

Theoretical approach.-Since accurate predictions of positron binding to polyatomic molecules are beyond the capacity of the best ab initio calculations, we use a modelcorrelation-potential approach [29]. The electrostatic potential $V_{\text {st }}$ of the molecule is calculated at the HartreeFock level using the standard $6-311++\mathrm{G}(d, p)$ basis, and then a potential that describes long-range polarization of the molecular electron cloud by the positron is added. The explicit form of this potential is

$V_{\text {cor }}(\mathbf{r})=-\sum_{A} \frac{\alpha_{A}}{2\left|\mathbf{r}-\mathbf{r}_{A}\right|^{4}}\left[1-\exp \left(-\left|\mathbf{r}-\mathbf{r}_{A}\right|^{6} / \rho_{A}^{6}\right)\right]$,

where the sum is over the atoms $A$ in the molecule, $\mathbf{r}$ is the position of the positron, and $\mathbf{r}_{A}$ is the position of nucleus $A$, relative to an arbitrary origin. [Atomic units (a.u.) are used throughout, unless stated otherwise.] This model potential uses the hybrid polarizabilities $\alpha_{A}$ of the molecule's constituent atoms [35], which take into account the chemical environment of the atom within the molecule. The factor in brackets provides a short-range cutoff, characterized by the cutoff radius $\rho_{A}$, which is a free parameter of the theory. Its values are expected to be 
comparable to the radii of the atoms involved, e.g., in the range of 1-3 a.u. Far from the molecule, the potential takes the asymptotic form $V_{\text {cor }}(\mathbf{r}) \simeq-\alpha / 2 r^{4}$, where $\alpha=\sum_{A} \alpha_{A}$ is the molecular polarizability [36]. The short-range part of the potential accounts for other important electron-positron correlation effects, such as virtual positronium formation.

The Schrödinger equation for the total potential $V_{\text {st }}+V_{\text {cor }}$ is solved to obtain the positron binding energy $\varepsilon_{b}$ and the positron wave function. In practice, this is done using the standard quantum-chemistry package GAMESS with the NEO plugin [37-40], which we have modified to include the model potential $V_{\text {cor }}$ [29]. We use an eventempered Gaussian basis consisting of $12 s$-type primitives centered on each $\mathrm{C}$ nucleus, with exponents $0.0001 \times 3^{i-1}$ $(i=1-12)$, and eight $s$-type primitives centered on each $\mathrm{H}$ nucleus, with exponents $0.0081 \times 3^{i-1}(i=1-8)$.

Binding energies for alkanes.-Here we apply the method to alkanes, which are nonpolar or very weakly polar molecules. While no quantum-chemistry calculations of positron binding have been reported for them before, positron binding energies have been measured for most of the $n$-alkanes $\mathrm{C}_{n} \mathrm{H}_{2 n+2}$ with $n=3-16$ (methane $\mathrm{CH}_{4}$ does not support a positron bound state, and while ethane $\mathrm{C}_{2} \mathrm{H}_{6}$ appears to bind a positron, $\varepsilon_{b}$ is too small to measure), and also for isopentane $\mathrm{C}_{5} \mathrm{H}_{12}$, cyclopropane $\mathrm{C}_{3} \mathrm{H}_{6}$, and cyclohexane $\mathrm{C}_{6} \mathrm{H}_{12}$ [41]. The binding energy for the $n$-alkanes was found to increase close to linearly with $n$, and a second bound state was observed for $n \geq 12$.

We choose $\alpha_{\mathrm{C}}=7.096$ and $\alpha_{\mathrm{H}}=2.650$ a.u., which provide the best fit, $\alpha=n \alpha_{\mathrm{C}}+(2 n+2) \alpha_{\mathrm{H}}$, of the polarizabilities of alkanes [35]. We use the same cutoff radius for the $\mathrm{C}$ and $\mathrm{H}$ atoms, and set $\rho_{A}=2.25$ a.u. to reproduce the measured $\varepsilon_{b}=220 \mathrm{meV}$ for dodecane $\mathrm{C}_{12} \mathrm{H}_{26}$. Figure 1 shows the values of $\varepsilon_{b}$ obtained for the $n$-alkanes $\mathrm{C}_{n} \mathrm{H}_{2 n+2}$ in terms of $n$. Also shown are the experimental data [41] and the crude zero-range-potential (ZRP) calculations (in which each of the $\mathrm{CH}_{2}$ or $\mathrm{CH}_{3}$ groups was replaced by a short-range deltalike potential, whose strength was chosen to fit the binding energy for dodecane) [42]. The present calculations and the experimental data are also shown in Table I. We obtain generally very good agreement with the experimental data. For $n=3-7$, our results follow the nearlinear trend of the experiment much more closely than do the zero-range-potential calculations. In particular, we report a positive binding energy for $n=3$ (propane), where the ZRP model shows no binding. Also, the present calculation predicts the emergence of a second bound state for $n=12$ (dodecane), in agreement with experiment, while the ZRP model only shows this for $n=13$. For $n=8$ (octane) and 9 (nonane), we observe a somewhat larger discrepancy with the measured binding energies. We note, however, that the experimental data for these molecules lie slightly below the linear trend set by the other molecules. This difference may therefore be due to an experimental error. From $n \approx 12$ upwards, the calculated

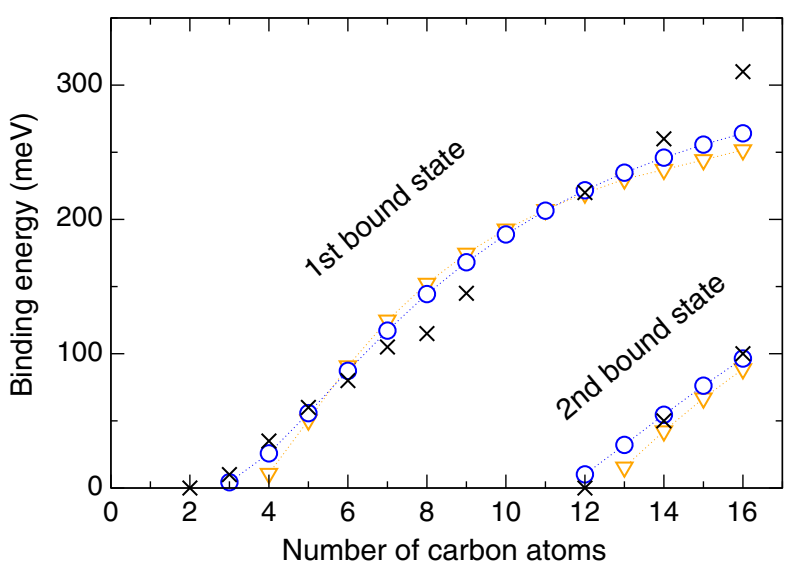

FIG. 1. Positron binding energies for $n$-alkane molecules $\mathrm{C}_{n} \mathrm{H}_{2 n+2}$. Black crosses, experiment [41]; blue circles, present calculation; orange triangles, zero-range potential model [42].

binding energies show signs of saturation and drop below the near-linear trend observed for smaller $n$; this effect is even more pronounced in the ZRP data. Indeed, for $n=14$ and 16 , our $\varepsilon_{b}$ for the first bound states underestimate the experimental values by $5 \%$ and $15 \%$, respectively, although the second bound state is still very well described. The exact reasons for this discrepancy are not clear. One

TABLE I. Calculated binding energies $\varepsilon_{b}$, independentparticle-approximation contact densities $\delta_{e p}^{(0)}$, and enhanced and renormalized contact densities $\delta_{e p}$ for $n$-alkane molecules $\mathrm{C}_{n} \mathrm{H}_{2 n+2}$. Also shown are the experimental (exp.) binding energies [41]. Square brackets indicate powers of 10 .

\begin{tabular}{lcccc}
\hline \hline & $\begin{array}{c}\varepsilon_{b} \\
n\end{array}$ & $\begin{array}{c}\varepsilon_{b}(\mathrm{exp} .) \\
(\mathrm{meV})\end{array}$ & $\begin{array}{c}\delta_{e p}^{(0)} \\
(\text { a.u. })\end{array}$ & $\begin{array}{c}\delta_{e p} \\
(\text { a.u. })\end{array}$ \\
\hline 2 & $-2.177^{\mathrm{a}}$ & $>0$ & & \\
3 & 4.302 & 10 & $5.717[-4]$ & $2.605[-3]$ \\
4 & 25.81 & 35 & $1.600[-3]$ & $7.221[-3]$ \\
5 & 55.75 & 60 & $2.547[-3]$ & $1.138[-2]$ \\
6 & 87.23 & 80 & $3.328[-3]$ & $1.476[-2]$ \\
7 & 117.2 & 105 & $3.948[-3]$ & $1.740[-2]$ \\
8 & 144.4 & 115 & $4.426[-3]$ & $1.943[-2]$ \\
9 & 168.1 & 145 & $4.791[-3]$ & $2.096[-2]$ \\
10 & 188.8 & & $5.068[-3]$ & $2.209[-2]$ \\
11 & 206.5 & & $5.280[-3]$ & $2.300[-2]$ \\
12 & 221.7 & 220 & $5.445[-3]$ & $2.367[-2]$ \\
$12^{\mathrm{b}}$ & 10.14 & 0 & $2.587[-3]$ & $1.158[-2]$ \\
13 & 234.8 & & $5.570[-3]$ & $2.420[-2]$ \\
$13^{\mathrm{b}}$ & 32.12 & & $3.337[-3]$ & $1.482[-2]$ \\
14 & 246.0 & 260 & $5.666[-3]$ & $2.458[-2]$ \\
$14^{\mathrm{b}}$ & 54.56 & 50 & $3.858[-3]$ & $1.703[-2]$ \\
15 & 255.7 & & $5.744[-3]$ & $2.492[-2]$ \\
$15^{\mathrm{b}}$ & 76.19 & & $4.257[-3]$ & $1.872[-2]$ \\
16 & 264.1 & 310 & $5.805[-3]$ & $2.516[-2]$ \\
$16^{\mathrm{b}}$ & 96.40 & 100 & $4.568[-3]$ & $2.004[-2]$ \\
\hline \hline
\end{tabular}

${ }^{a}$ With no binding, this value is determined by the size of the basis.

${ }^{\mathrm{b}}$ Second bound state. 

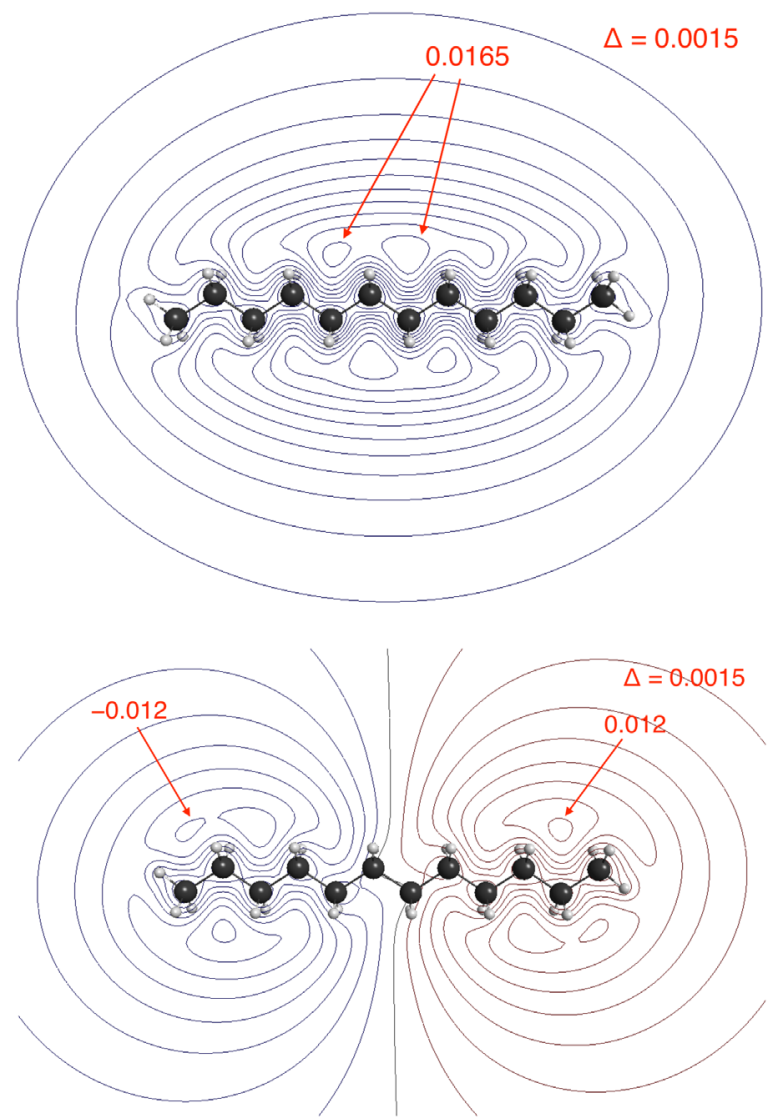

FIG. 2. Contour plots of the first (upper panel) and second (lower panel) bound positron states for dodecane $\mathrm{C}_{12} \mathrm{H}_{26}$. The contour for which the magnitude of the wave function is largest is indicated. The change in the magnitude of the wave function between neighboring contours is $\Delta=0.0015$.

possibility is that at room temperatures such large chain molecules may favor conformations other than linear [43], for which the calculations were performed. At the other end of the scale, our calculations with $\rho_{A}=2.25$ a.u. fail to predict a bound state for $n=2$ (ethane), and it would be necessary to reduce the value of the cutoff radius to 2.09 a.u. for a bound state to appear. This likely reflects the fact that the cutoff radius can have a weak dependence on the size of the molecule, which becomes more obvious for smaller species.

Figure 2 shows the shapes of the first and second bound positron orbitals for dodecane. We see that the positron cloud surrounds the entire molecule, as was inferred from the analysis of measured annihilation $\gamma$-ray spectra [44]. This is in contrast to strongly polar molecules, where the bound positron is strongly localized around the negative end of the dipole $[12,29]$. The wave function of the second bound state has a $p$-wave character. It changes sign when crossing a nodal surface ("plane") near the center of the molecule.

Besides the near-linear increase of the binding energy for $n$-alkanes, the experiment found that isopentane $\mathrm{C}_{5} \mathrm{H}_{12}$, cyclopropane $\mathrm{C}_{3} \mathrm{H}_{6}$, and cyclohexane $\mathrm{C}_{6} \mathrm{H}_{12}$ have the same binding energies as the $n$-alkanes with the same number of carbon atoms [41]. Using our method, we find that the binding energy for isopentane is $\varepsilon_{b}=59 \mathrm{meV}$, which is only $5 \%$ greater than the calculated value of $56 \mathrm{meV}$ for $n$ pentane. Both values are close to the experimental value $\varepsilon_{b}=60 \mathrm{meV}$ [41]. (The accuracy of the experimental determination of $\varepsilon_{b}$ is likely no better than $5 \mathrm{meV}$, due to uncertainties in the energy of the positron beam.) For neopentane, our calculations yield $\varepsilon_{b}=57 \mathrm{meV}$, though there are no measurements for this isomer. The similarity between the binding energies for the three isomers suggests the long-range behavior of $V_{\text {cor }}$ (which is the same in all three cases) is more important for positron binding than the effects of the molecular geometry. The calculated values for cyclopropane and $n$-propane are $\varepsilon_{b}=0.66$ and $4.3 \mathrm{meV}$, respectively, while the experimental value is $10 \mathrm{meV}$. The smaller calculated binding energy for cyclopropane is due to the fact that its polarizability is $12 \%$ smaller than that of $n$-propane. Similarly, the calculated binding energies for cyclohexane and $n$-hexane are 76 and $87 \mathrm{meV}$, respectively, which can be attributed to the $7 \%$ smaller polarizability of cyclohexane. Experimentally, they were reported to have same binding energy of $80 \mathrm{meV}$ [41]. However, updated analysis using a somewhat higher resolution beam indicates $\varepsilon_{b}=80 \mathrm{meV}$ for cyclohexane and $\varepsilon_{b}=95 \mathrm{meV}$ for $n$ hexane [45], in close accord with the calculations.

Annihilation rates for alkanes.-The $2 \gamma$ annihilation rate for the positron from the bound state, for a system with a zero total electron spin, is given by $\Gamma=\pi r_{0}^{2} c \delta_{e p}$, where $r_{0}$ is the classical electron radius, $c$ is the speed of light, and $\delta_{e p}$ is the electron-positron contact density in the bound state [1]. A useful conversion from the contact density to the annihilation rate is $\Gamma\left[\mathrm{ns}^{-1}\right]=50.470 \times \delta_{e p}[$ a.u. $]$. The lifetime of the positron-molecule complex with respect to annihilation is $1 / \Gamma$.

We use the wave functions of the electronic molecular orbitals along with the positron wave function to calculate the electron-positron contact density $\delta_{e p}$, viz.,

$$
\delta_{e p}=\sum_{i} \gamma_{i} \int\left|\varphi_{i}(\mathbf{r})\right|^{2}|\psi(\mathbf{r})|^{2} d \tau,
$$

where the sum is over all of the occupied Hartree-Fock electronic spin orbitals with wave functions $\varphi_{i}, \psi$ is the positron wave function, and $\gamma_{i}$ is an annihilation vertex enhancement factor, specific to spin orbital $i$. The enhancement factor is introduced to improve on the independentparticle approximation by accounting for an increase of the electron density at the positron due to their Coulomb interaction [46]. Similar enhancement factors are used in calculations of positron annihilation in solids [47,48]. Recent many-body-theory calculations for atoms have shown that the enhancement factors are, to a good approximation, functions of the spin-orbital energy $\varepsilon_{i}[29,46]$ : 


$$
\gamma_{i}=1+\sqrt{\frac{1.31}{-\varepsilon_{i}}}+\left(\frac{0.834}{-\varepsilon_{i}}\right)^{2.15} .
$$

We also renormalize the positron wave function, to take into account the underlying many-body nature of $V_{\text {cor }}$. The true correlation potential that describes the interaction of a positron with a many-electron system is a nonlocal and energy-dependent operator $\Sigma_{E}\left(\mathbf{r}, \mathbf{r}^{\prime}\right)[49,50]$. When using it in the Schrödinger-like Dyson equation, the negativeenergy eigenvalue $\varepsilon_{0}=-\varepsilon_{b}$ that corresponds to a bound state becomes a function of $E$, i.e., $\varepsilon_{0}=\varepsilon_{0}(E)$ and has to be found self-consistently. The corresponding positron wave function is, in fact, a quasiparticle wave function, normalized as [51,52]

$$
\int|\psi(\mathbf{r})|^{2} d \tau=\left(1-\frac{\partial \varepsilon_{0}}{\partial E}\right)^{-1} \equiv a<1 .
$$

By considering the dependence of the binding energy on the molecular polarizability, we have determined values of $a$ for each molecule. The values range from $a=0.992$ for $\mathrm{C}_{3} \mathrm{H}_{8}$ to 0.933 for $\mathrm{C}_{16} \mathrm{H}_{34}$, for the first bound state, and from $a=0.967$ for $\mathrm{C}_{12} \mathrm{H}_{26}$ to 0.946 for $\mathrm{C}_{16} \mathrm{H}_{34}$, for the second bound state.

Figure 3(a) shows the contact density for each of the $n$ alkanes, for the first and second bound states, when the latter exists. Results are shown for the independent-particle approximation $\left(\gamma_{i}=1\right.$ and $\left.a=1\right)$, and also with enhancement and renormalization, i.e., using Eqs. (3) and (4). These data are also shown in Table I. Including the enhancement factors and renormalization increases the contact density by a factor of approximately 4.5 compared to the independent-particle approximation, irrespective of the size of the molecule. The growth of the contact density with the size of the molecule is related to an increase in the positron binding energy. Previous studies of positron-atom bound states found that the contact density grew linearly with $\sqrt{\varepsilon_{b}}$, specifically, as $\delta_{e p} \approx 9.0 \times 10^{-4} \sqrt{\varepsilon_{b}}$, where $\delta_{e p}$ is in a.u. and $\varepsilon_{b}$ is in $\mathrm{meV}[1,3]$. This dependence is related to the probability of finding the positron in the vicinity of the target for weakly bound $s$-type states. Figure 3(b) shows that the contact density for the $n$-alkanes also scales linearly with $\sqrt{\varepsilon_{b}}$, with $\delta_{e p}^{(0)} \approx 3.63 \times 10^{-4} \sqrt{\varepsilon_{b}}$ in the independentparticle approximation (thin black dashed line), and $\delta_{e p} \approx 1.58 \times 10^{-3} \sqrt{\varepsilon_{b}}$, when the enhancement factors and renormalization are included (thin blue dashed line). Thus we see that the contact densities for positron bound states with alkanes are about 1.8 times greater than those for the positron-atom bound states, for the same binding energy. This difference must be related to the fact that in atoms, positron access of high-electron-density regions is always impeded by the nuclear repulsion, while in molecules it is easier for the positron to approach the electrons as they are shared between the constituent atoms. It is also
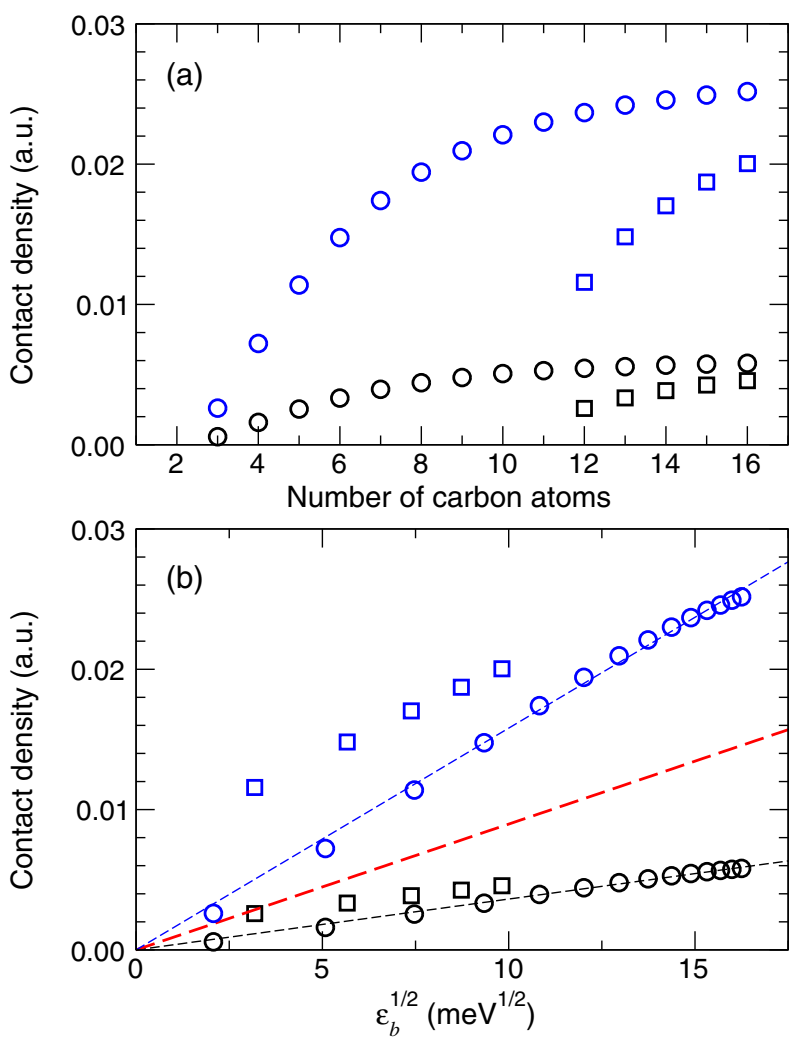

FIG. 3. Electron-positron contact density for $n$-alkane molecules $\mathrm{C}_{n} \mathrm{H}_{2 n+2}$. Panel (a) shows the contact density in terms of $n$, while panel (b) shows it as a function of $\sqrt{\varepsilon_{b}}$. Black symbols, independent-particle approximation; blue symbols, with enhancement factors and renormalization. Circles, first bound state; squares, second bound state. In (b), thin black and blue dashed lines are fits of the respective first-bound-state data, and the thick red dashed line is a fit of the calculated contact densities for positron-atom bound states [1].

worth noting that the contact density for the second bound state remains finite when its binding energy goes to zero. Such behavior is characteristic of $p$-type states that remain localized in the limit $\varepsilon_{b} \rightarrow 0$.

We have also calculated contact densities for the isomers of pentane, cyclopropane, and cyclohexane. The values that include the enhancement factors and renormalization are $1.3 \times 10^{-3}$ for cyclopropane, $1.2 \times 10^{-2}$ for isopentane and neopentane, and $1.4 \times 10^{-2}$ a.u. for cyclohexane. With the exception of cyclopropane, the contact densities for the various isomers and ring forms are very close to those for the corresponding $n$-alkane in Table I. For cyclopropane, the contact density is half that of $n$-propane. This is related to the fact that the calculated binding energy for cyclopropane is 6 times smaller than that of $n$-propane.

Summary.-We have developed a method for calculating positron-molecule binding energies and annihilation rates and demonstrated its predictive capabilities for the alkanes. These quantities are key to understanding positron resonant annihilation in molecules. Our method allows one to 
investigate positron binding to other molecules that have been studied experimentally. It can also be used to make predictions for other molecular species, to guide future experimental effort and provide comparisons for more sophisticated quantum-chemistry calculations. The positron wave function can also be used to calculate the annihilation $\gamma$ spectra, where much of the experimental data [44] still await theoretical analysis [53].

We are very grateful to J. R. Danielson, S. Ghosh, and C. M. Surko for providing recent unpublished experimental data. This work has been supported by the EPSRC UK, Grant No. EP/R006431/1.

*a.swann@qub.ac.uk

†.gribakin@qub.ac.uk

[1] G. F. Gribakin, J. A. Young, and C. M. Surko, Positronmolecule interactions: Resonant attachment, annihilation, and bound states, Rev. Mod. Phys. 82, 2557 (2010).

[2] G. F. Gribakin, Mechanisms of positron annihilation on molecules, Phys. Rev. A 61, 022720 (2000).

[3] G. F. Gribakin, Theory of positron annihilation on molecules, in New Directions in Antimatter Chemistry and Physics, edited by C. M. Surko and F. A. Gianturco (Kluwer Academic, Dordrecht, 2001), Chap. 22.

[4] M. Deutsch, Three-quantum decay of positronium, Phys. Rev. 83, 866 (1951).

[5] D. A. L. Paul and L. Saint-Pierre, Rapid Annihilations of Positrons in Polyatomic Gases, Phys. Rev. Lett. 11, 493 (1963).

[6] G. R. Heyland, M. Charlton, T. C. Griffith, and G. L. Wright, Positron lifetime spectra for gases, Can. J. Phys. 60, 503 (1982).

[7] C. M. Surko, A. Passner, M. Leventhal, and F. J. Wysocki, Bound States of Positrons and Large Molecules, Phys. Rev. Lett. 61, 1831 (1988).

[8] K. Iwata, R. G. Greaves, T. J. Murphy, M. D. Tinkle, and C. M. Surko, Measurements of positron-annihilation rates on molecules, Phys. Rev. A 51, 473 (1995).

[9] S. J. Gilbert, L. D. Barnes, J. P. Sullivan, and C. M. Surko, Vibrational-Resonance Enhancement of Positron Annihilation in Molecules, Phys. Rev. Lett. 88, 043201 (2002).

[10] J. R. Danielson, J. A. Young, and C. M. Surko, Dependence of positron-molecule binding energies on molecular properties, J. Phys. B 42, 235203 (2009).

[11] J. R. Danielson, J. J. Gosselin, and C. M. Surko, Dipole Enhancement of Positron Binding to Molecules, Phys. Rev. Lett. 104, 233201 (2010).

[12] J. R. Danielson, A. C. L. Jones, M. R. Natisin, and C. M. Surko, Comparisons of Positron and Electron Binding to Molecules, Phys. Rev. Lett. 109, 113201 (2012).

[13] J. R. Danielson, A. C. L. Jones, J. J. Gosselin, M. R. Natisin, and C.M. Surko, Interplay between permanent dipole moments and polarizability in positron-molecule binding, Phys. Rev. A 85, 022709 (2012).

[14] D. M. Schrader, Compounds or positrons with koino-atoms and -molecules, in Proceedings of the International School of Physics "Enrico Fermi," Course CLXXIV "Physics with
Many Positrons", edited by R. S. Brusa, A. Dupasquier, and A. P. Mills, Jr. (IOS Press, Amsterdam, 2010), pp. 337-398.

[15] M. Tachikawa, I. Shimamura, R. J. Buenker, and M. Kimura, Bound states of positron with molecules, in New Directions in Antimatter Chemistry and Physics, edited by C. M. Surko and F. A. Gianturco (Kluwer Academic, Dordrecht, 2001), Chap. 23.

[16] A static molecule with a dipole moment greater than 1.625 D will always bind an electron or positron [17], while for a molecule that is free to rotate, the critical value dipole moment increases with the angular momentum of the molecule [18].

[17] O. H. Crawford, Bound states of a charged particle in a dipole field, Proc. Phys. Soc. 91, 279 (1967).

[18] W. R. Garrett, Critical binding of an electron to a rotationally excited dipolar system, Phys. Rev. A 3, 961 (1971).

[19] H. A. Kurtz and K. D. Jordan, Theoretical studies of positron-molecule complexes, J. Chem. Phys. 75, 1876 (1981).

[20] K. Strasburger, Quantum chemical study on complexes of the $\mathrm{LiH}$ molecule with $e^{+}$, Ps and $\mathrm{Ps}^{-}$including correlation energy, Chem. Phys. Lett. 253, 49 (1996).

[21] H. Chojnacki and K. Strasburger, Configuration interaction study of the positronic hydrogen cyanide molecule, Mol. Phys. 104, 2273 (2006).

[22] F. A. Gianturco, J. Franz, R. J. Buenker, H.-P. Liebermann, L. Pichl, J.-M. Rost, M. Tachikawa, and M. Kimura, Positron binding to alkali-metal hydrides: The role of molecular vibrations, Phys. Rev. A 73, 022705 (2006).

[23] D. Bressanini, M. Mella, and G. Morosi, Positron chemistry by quantum Monte Carlo. II. Ground-state of positron-polar molecule complexes, J. Chem. Phys. 109, 1716 (1998).

[24] M. Mella, G. Morosi, D. Bressanini, and S. Elli, Positron and positronium chemistry by quantum Monte Carlo. V. The ground state potential energy curve of $e^{+} \mathrm{LiH}, \mathrm{J}$. Chem. Phys. 113, 6154 (2000).

[25] Y. Kita, R. Maezono, M. Tachikawa, M. Towler, and R. J. Needs, $\mathrm{Ab}$ initio quantum Monte Carlo study of the positronic hydrogen cyanide molecule, J. Chem. Phys. 131, 134310 (2009).

[26] S. Bubin and L. Adamowicz, Non-Born-Oppenheimer study of positronic molecular systems: $e^{+} \mathrm{LiH}, \mathrm{J}$. Chem. Phys. 120, 6051 (2004).

[27] J. Romero, J. A. Charry, R. Flores-Moreno, M. T. do N. Varella, and A. Reyes, Calculation of positron binding energies using the generalized any particle propagator theory, J. Chem. Phys. 141, 114103 (2014).

[28] K. Koyanagi, Y. Takeda, T. Oyamada, Y. Kita, and M. Tachikawa, Positron-attachment to nonpolar or small dipole $\mathrm{CXY}(\mathrm{X}, \mathrm{Y}=\mathrm{O}, \mathrm{S}$, and Se) molecules: Vibrational enhancement of positron affinities with configuration interaction level of multi-component molecular orbital approach, Phys. Chem. Chem. Phys. 15, 16208 (2013).

[29] A. R. Swann and G. F. Gribakin, Calculations of positron binding and annihilation in polyatomic molecules, J. Chem. Phys. 149, 244305 (2018).

[30] M. Tachikawa, Y. Kita, and R. J. Buenker, Bound states of the positron with nitrile species with a configuration interaction multi-component molecular orbital approach, Phys. Chem. Chem. Phys. 13, 2701 (2011). 
[31] M. Tachikawa, Y. Kita, and R. J. Buenker, Bound states of positron with simple carbonyl and aldehyde species with configuration interaction multi-component molecular orbital and local vibrational approaches, New J. Phys. 14, 035004 (2012).

[32] M. Tachikawa, Positron-attachment to acetonitrile, acetaldehyde, and acetone molecules: Vibrational enhancement of positron affinities with configuration interaction level of multi-component molecular orbital approach, J. Phys. Conf. Ser. 488, 012053 (2014).

[33] Calculations for $\mathrm{CS}_{2}$ [28] gave a negative binding energy, vs measured $\varepsilon_{b}=75 \mathrm{meV}$ [11]. Scattering calculations for allene indicate binding [34], but no estimate of $\varepsilon_{b}$ is provided.

[34] A. S. Barbosa, S. A. Sanchez, and M. H. F. Bettega, Bound state in positron scattering by allene, Phys. Rev. A 96, 062706 (2017).

[35] K. J. Miller, Additivity methods in molecular polarizability, J. Am. Chem. Soc. 112, 8533 (1990).

[36] The present implementation regards atomic contributions to $V_{\text {cor }}(\mathbf{r})$ as spherically symmetric. In future, they can be made anisotropic, to correlate with the directions of adjacent bonds.

[37] M. W. Schmidt, K. K. Baldridge, J. A. Boatz, S. T. Elbert, M. S. Gordon, J. H. Jensen, S. Koseki, N. Matsunaga, K. A. Nguyen, S. J. Su, T. L. Windus, M. Dupuis, and J. A. Montgomery, General atomic and molecular electronic structure system, J. Comput. Chem. 14, 1347 (1993).

[38] M. S. Gordon and M. W. Schmidt, Advances in electronic structure theory, in Theory and Applications of Computational Chemistry: The First Forty Years, edited by C. E. Dykstra, G. Frenking, K. S. Kim, and G. E. Scuseria (Elsevier, Amsterdam, 2005), Chap. 41.

[39] S. P. Webb, T. Iordanov, and S. Hammes-Schiffer, Multiconfigurational nuclear-electronic orbital approach: Incorporation of nuclear quantum effects in electronic structure calculations, J. Chem. Phys. 117, 4106 (2002).

[40] P. E. Adamson, X. F. Duan, L. W. Burggraf, M. V. Pak, C. Swalina, and S. Hammes-Schiffer, Modeling positrons in molecular electronic structure calculations with the nuclearelectronic orbital method, J. Phys. Chem. A 112, 1346 (2008).
[41] J. A. Young and C.M. Surko, Feshbach-resonancemediated annihilation in positron interactions with large molecules, Phys. Rev. A 77, 052704 (2008).

[42] G. F. Gribakin and C. M. R. Lee, Positron annihilation in large polyatomic molecules. The role of vibrational Feshbach resonances and binding, Eur. Phys. J. D 51, 51 (2009).

[43] L. L. Thomas, T. J. Christakis, and W. L. Jorgensen, Conformation of alkanes in the gas phase and pure liquids, J. Phys. Chem. B 110, 21198 (2006).

[44] K. Iwata, R. G. Greaves, and C. M. Surko, $\gamma$-ray spectra from positron annihilation on atoms and molecules, Phys. Rev. A 55, 3586 (1997).

[45] J. R. Danielson, S. Ghosh, and C. M. Surko (private communication).

[46] D. G. Green and G. F. Gribakin, $\gamma$-Ray Spectra and Enhancement Factors for Positron Annihilation with Core Electrons, Phys. Rev. Lett. 114, 093201 (2015).

[47] M. J. Puska and R. M. Nieminen, Theory of positrons in solids and on solid surfaces, Rev. Mod. Phys. 66, 841 (1994).

[48] M. Alatalo, B. Barbiellini, M. Hakala, H. Kauppinen, T. Korhonen, M. J. Puska, K. Saarinen, P. Hautojärvi, and R. M. Nieminen, Theoretical and experimental study of positron annihilation with core electrons in solids, Phys. Rev. B 54, 2397 (1996).

[49] G. F. Gribakin and J. Ludlow, Many-body theory of positron-atom interactions, Phys. Rev. A 70, 032720 (2004).

[50] D. G. Green, J. A. Ludlow, and G. F. Gribakin, Positron scattering and annihilation on noble-gas atoms, Phys. Rev. A 90, 032712 (2014).

[51] L. V. Chernysheva, G. F. Gribakin, V. K. Ivanov, and M. Y. Kuchiev, Many-body calculation of negative ions using the Dyson equation, J. Phys. B 21, L419 (1988).

[52] J. A. Ludlow and G. F. Gribakin, Many-body theory calculations of positron binding to negative ions, Int. Rev. At. Mol. Phys. 1, 73 (2010); arXiv:1002.3125.

[53] Y. Ikabata, R. Aiba, T. Iwanade, H. Nishizawa, F. Wang, and H. Nakai, Quantum chemical approach for positron annihilation spectra of atoms and molecules beyond plane-wave approximation, J. Chem. Phys. 148, 184110 (2018). 